\title{
ORIGINAL ARTICLE Impact of type 1 diabetes mellitus and celiac disease on nutrition and quality of life
}

\author{
JG Nunes-Silva ${ }^{1}$, VS Nunes ${ }^{2}$, RP Schwartz ${ }^{3}$, S MLSS Trecco ${ }^{1}$, D Evazian ${ }^{1}$, ML Correa-Giannella ${ }^{4}$, M Nery $^{5}$ and MS Queiroz ${ }^{5}$
}

OBJECTIVE: Type 1 diabetes mellitus (T1DM) and celiac disease (CD) are autoimmune diseases and have similar genetic patterns. T1DM treatment is based on diet, physical activity and insulin therapy, whereas CD depends on dietary changes with restriction of wheat, rye and barley. The aim of the study was to evaluate the quality of life (QoL) of individuals with the association of T1DM and $C D$, to characterize their nutritional status and to compare it with those with only one disease and healthier controls.

SUBJECTS/METHODS: Sixty patients controlled by sex, age and body mass index (BMI) were stratified by previous diagnosis in: T1DM and CD (DMCD group); T1DM (DM group); CD (CD group); or healthy participants (HC). The SF-36 questionnaire was applied to assess psychological well being and results were compared with glycemic control and presence of complications related to diabetes, adhesion to gluten-free diet (GFD). Nutritional status and body mass composition were determined by BMI, waist circumference, bioimpedance, general laboratory tests and whole-body densitometry.

RESULTS: The time of diagnosis of T1DM was similar between DMCD and DM groups; however, the duration of CD was significantly higher in the CD group compared with DMCD. The SF-36 analysis revealed statistically significant differences between DM and HC groups in two domains: general health $(P=0.042)$ and energy/vitality $(P=0.012)$. QoL was also correlated with compliance to a GFD, and scores were similar in both groups: DMCD and CD. Forty percent of individuals in the CD group had visceral fat area above $100 \mathrm{~cm}^{2}$, as opposed to $20 \%$ in the other groups.

CONCLUSIONS: Individuals of DMCD group had similar scores to DM, CD and HC on QoL, as well as on their nutritional status and bone metabolism. Thereby, we should conclude that the association of T1DM and CD did not deteriorate their health status.

Nutrition \& Diabetes (2017) 7, e239; doi:10.1038/nutd.2016.43; published online 9 January 2017

\section{INTRODUCTION}

Type 1 diabetes mellitus (T1DM) and celiac disease (CD) are autoimmune diseases caused by the interactions of genetic and environmental factors. Both diseases have genetic patterns linked to HLA-DQ2 and HLA-DQ8, resulting in a reported prevalence of $C D$ in T1DM five to seven times higher than in the general population. ${ }^{1}$ T1DM treatment is based on the regular physical activity, and nutritional and insulin therapy. ${ }^{2}$ Hence, the medical prescription of multiple doses of insulin and carbohydrate counting showed better glycemic control in individuals with DM1, avoiding the glucose restriction and the negative impact of dietary restriction. ${ }^{3}$

Similarly, treatment for CD is based on the dietary therapy, with restriction of wheat, rye and barley, responsible for the immune system activation and intestinal damage. Proper gluten restriction results in a recovery of the $C D$ enteropathy, despite conflicting results about the adequacy of daily requirements of micronutrients and macronutrients. The appropriateness of nutrients is related to the availability, composition of gluten-free food, cultural aspects, access to 'new diets' and specific nutritional recommendations for each population. ${ }^{4-6}$ Overall, individuals with adequate adhesion to the gluten-free diet (GFD) tend to consume smaller amounts of fibers, iron, calcium, folic acid and vitamin B12.,

Some studies have shown an improvement in quality of life scores (QoL) for those patients with T1DM after initiating carbohydrate counting and intensive insulin therapy, but the need for full-time attention to the disease for a long time may deteriorate their QoL., ${ }^{9,10}$ Initially, the QoL of celiac patients is affected by diet restriction; however, it improves $\sim 1$ year after the introduction of GFD, even in those with a partial adherence. ${ }^{11,12}$ Despite the high prevalence of the association between T1DM and $C D$, the repercussion of dietary changes imposed by both diseases on QoL has been poorly evaluated. Therefore, the aim of the study was to evaluate the QoL of individuals with the association of T1DM and CD, and to characterize their nutritional status, nutritional behavior and deficiencies, comparing it with those with only one disease (T1DM or CD) and healthy controls

\section{PATIENTS AND METHODS}

\section{Patients}

To evaluate the nutritional status, and characterize usual dietary intake and impact on QoL of individuals with T1DM associated with CD, without biases related to sex, age and body mass index (BMI), we used the parameters of the 15 patients enrolled in the DMCD group to match with those of participants from the other three groups. Sixty patients controlled by sex, age and BMI were stratified by previous diagnosis in four groups: T1DM and CD (DMCD group); T1DM (DM group); CD (CD group); or healthy participants (HC group). They were recruited from Hospital das Clinicas da Faculdade de Medicina, Universidade São Paulo, endocrinology and gastroenterology outpatient clinics, although healthy participants were

\footnotetext{
${ }^{1}$ Nutrition and Dietetics Division, Central Institute of Clinics Hospital, University of São Paulo Medical School, São Paulo, Brazil; ${ }^{2}$ Lipids Laboratory (LIM-10), Endocrinology and Metabolism Division of Hospital das Clinicas, Faculty of Medical Sciences, University of Sao Paulo, São Paulo, Brazil; ${ }^{3}$ Radiology Institute of Clinics Hospital, University of São Paulo Medical School, São Paulo, Brazil; ${ }^{4}$ Cellular and Molecular Endocrinology Laboratory (LIM-18), University of São Paulo Medical School, São Paulo, Brazil and ${ }^{5}$ Endocrinology

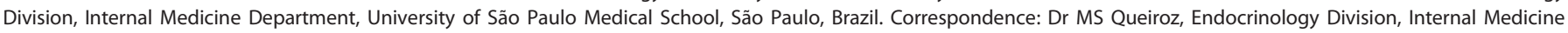
Department, University of São Paulo Medical School, Av. Dr Enéas Carvalho de Aguiar, 155 - 8o. andar - Bloco 8A - Setor Azul, São Paulo 05403-000, Brazil. 
recruited from the nutrition and dietetics division. We adopted as inclusion criteria: (1) adult individuals aged 18-59 years; (2) type 1 diabetes diagnosed by presence of antibodies anti-glutamic acid decarboxylase or anti-islet or C-peptide $<0.5 \mathrm{ng} \mathrm{ml}^{-1}$ or dependence on insulin since diagnosis of the disease; (3) CD confirmed by antibodies, anti-endomysial antibodies (anti-EMA) or anti-transglutaminase antibody positives and typical histologic alterations on intestinal biopsy. The Institutional Review Board at Hospital das Clinicas approved these studies; all participants provided signed, informed consent. All patients with diabetes were on intensive multiple doses insulin therapy, just one patient at DMCD group was with continuous subcutaneous insulin infusion. The average dose of insulin was $0.69 \pm 0.37$ and $0.61 \pm 0.19$ units per $\mathrm{kg}$ per day, group DMCD and $C D$, respectively.

Nutritional status and body mass composition

The area of visceral fat was measured using octopolar bioimpedance (InBody720, Biospace Co. Ltd., South Korea), whereas total body fat and bone mineral density were assessed by whole-body densitometry (dual energy X-ray absorptiometry, Hologic Discovery W; Bedford, MA 01730-1401, USA). The registered dietitian nutritionist responsible for the project execution plan (Joyce Gouveia Nunes-Silva-S) determined nutritional status by BMI and waist circumference, assessed food intake by a 3day food record and calculated nutritional composition using the Virtual Nutri Plus software (Copyright 2012-www.keeple.com.br) and the Brazilian Food Composition Table, TACO ${ }^{13}$ and Philippi. ${ }^{14}$ Results of macronutrients, fibers and cholesterol were compared with the recommendations of the Brazilian Society of Diabetes, ${ }^{15}$ and micronutrients to the references of the Institute of Medicine. ${ }^{16,17}$

\section{Psychological well-being assessment}

We applied the Brazilian version of the SF-36 questionnaire ${ }^{18}$ and compared data on QoL among all four groups. The value for each domain of SF-36 ranges from 0 to 100 ( 0 being the worst and 100, the best state of health). For groups with T1DM, SF-36 results were also associated with glycemic control and presence of complications related to diabetes, whereas for those with $C D$, adhesion to diet assessed by Celiac Dietary Adherence Test (CDAT) ${ }^{19}$ and anti-EMA were used for comparison. To infer the degree of commitment to the GFD, we used as criterion for CDAT: good-diet adhesion, scores $<13$, low-diet adhesion and scores $>17$, as suggested by the authors. ${ }^{19}$ A single investigator administered both questionnaires, SF-36 and CDAT during the interview for nutritional assessment.

\section{Laboratory methods}

Anti-EMA were detected by indirect immunofluorescence against human umbilical cord, adopting $<1 / 10$ as cutoff. Glycated hemoglobin (normal range 4-6\%) was determined in whole blood using ion-exchange highperformance liquid chromatography. General laboratory tests and other biochemical analyses were carried out using commercial kits, as part of the routine assessment after overnight fasting.

\section{Statistical analyses}

We used Excel 2011 and SPSS version 20.0 (IBM SPSS Statistics for Windows, IBM Corp., Armonk, NY, USA) for statistical analysis. Variables were described as mean and s.d., median and range (minimum and maximum). The analysis of variance test was applied to analyze variance, whereas normality of data distribution was assumed within each group using the Kolmogorov-Smirnov test. For variables with statistically significant differences between groups, we performed the Bonferroni test for multiple comparisons. We applied the non-parametric test of Fisher and Pearson correlation coefficient to analyze independent variables, and to assess the relationship between the variables, respectively. Tests were performed with a significance level of $5 \%$.

\section{RESULTS}

Clinical details are listed in Table 1 and are equal regarding age, sex and BMI in all groups. The duration of T1DM was similar between DMCD and DM groups; however, the duration of CD was significantly higher in the $C D$ group compared to DMCD $(P=0.0015)$.
As shown in Figure 1, mean visceral fat area and body fat percentages were similar among all groups. Yet, $50 \%$ of women in the $C D$ group had visceral fat area above $100 \mathrm{~cm}^{2}$, whereas only $33 \%$ of patients achieved this cutoff in the other groups (Table 2 ). In general, $64 \%$ of women participants were considered as having increased risk for metabolic complications, according to the criteria of the World Health Organization. ${ }^{20}$ On the other hand, all

Table 1. Demographic characteristics of the patients enrolled in the study according to groups

\begin{tabular}{|c|c|c|c|c|c|}
\hline Groups & $\begin{array}{l}D M C D \\
(\mathrm{n}=15)\end{array}$ & $\begin{array}{c}D M \\
(\mathrm{n}=15)\end{array}$ & $\begin{array}{c}C D \\
(\mathrm{n}=15)\end{array}$ & $\begin{array}{c}H C \\
(n=15)\end{array}$ & P-value \\
\hline Age (years) & $37.4 \pm 13.4$ & $35.5 \pm 12.5$ & $38 \pm 11.6$ & $36.8 \pm 12.4$ & 0.955 \\
\hline BMI $\left(\mathrm{kg} \mathrm{m}^{-2}\right)$ & $23.2 \pm 3.0$ & $24.0 \pm 3.1$ & $22.9 \pm 3.6$ & $23.1 \pm 2.7$ & 0.794 \\
\hline $\operatorname{Sex}(M / F)$ & $3 / 12$ & $3 / 12$ & $3 / 12$ & $3 / 12$ & \\
\hline $\begin{array}{l}\text { Duration } \\
\text { T1DM (years) }\end{array}$ & $19.8 \pm 9.4$ & $22.2 \pm 9.7$ & & & $0.493^{*}$ \\
\hline $\begin{array}{l}\text { Duration CD } \\
\text { (years) }\end{array}$ & $6.4 \pm 3.7$ & & $12.2 \pm 9.2$ & & $0.0015^{*}$ \\
\hline
\end{tabular}

Abbreviations: $\mathrm{BMI}$, body mass index; $\mathrm{CD}$, celiac disease; $\mathrm{DM}$, type 1 diabetes mellitus; $D M C D$, type 1 diabetes mellitus and celiac disease; $\mathrm{HC}$, healthy control; M/F, male/female. ${ }^{*} P$-value: ANOVA or Student's $t$-test. Values are means \pm s.d.
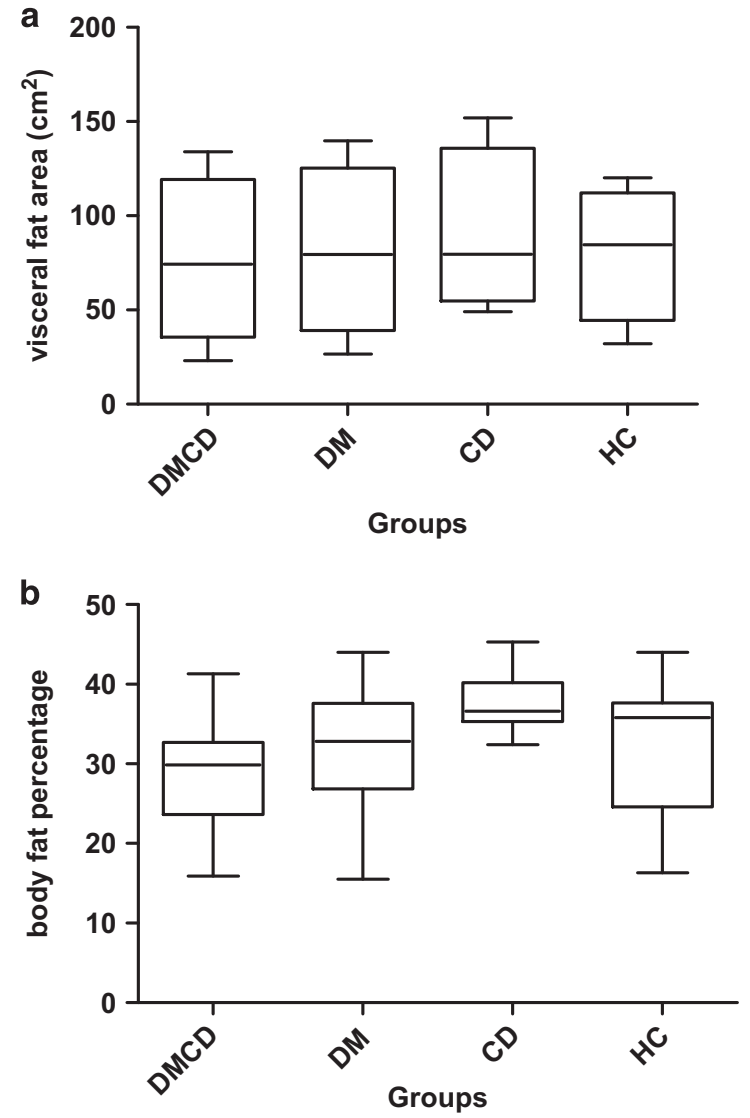

Figure 1. Visceral fat area and percentage of body fat according to dual energy X-ray absorptiometry (DEXA) among all four different groups. CD, celiac disease only; DM, type 1 diabetes only; DMDC, type 1 diabetes mellitus and celiac disease; $\mathrm{HC}$, healthy control. Values are expressed as means \pm s.d., median and range (minimum and maximum). (a) Visceral fat area $\left(\mathrm{cm}^{2}\right)$ and (b) body fat percentage. 
male patients had BMI $<25 \mathrm{~kg} \mathrm{~m}^{-2}$, waist circumference $<94 \mathrm{~cm}$ and visceral fat $<100 \mathrm{~cm}^{2}$. Taken together, these data pointed to similarities of clinical characteristics between groups and the tendency of women with $C D$ to have higher risk of metabolic complications.

All participants had normocaloric habitual food intake with nutritional composition appropriately distributed by macronutrients according to standards of the Institute of Medicine ${ }^{16,17}$ for the adult population. The quality of diets was adequate for carbohydrates (except for the DM group) and protein, but hyperlipidemic (except for HC). The consumption of B12 vitamin and selenium was satisfactory, whereas fiber, calcium and vitamin $D$ intake did not reach the daily recommendations in all groups. Laboratory measurements of vitamins and minerals were similar in all groups, and within the normal range (Table 3). Nonetheless, serum levels of folic acid and magnesium were lower in individuals with $C D$ (DMCD and $C D$ groups) and patients with diabetes (DMCD and DM groups), respectively (Table 4). In general, it is possible to conclude that having DM1, CD or both diseases did not affect the quality of food ingested in all groups.

We also assessed bone metabolism as shown in Table 3. Our data show that all laboratorial parameters measured were similar in all groups, with only three patients with $T$-score compatible

Table 2. Body mass composition of the women patients enrolled in the study according to groups

\begin{tabular}{lccccc}
\hline Groups & $\begin{array}{c}D M C D \\
(\mathrm{n}=12)\end{array}$ & $\begin{array}{c}D M \\
(\mathrm{n}=12)\end{array}$ & $\begin{array}{c}C D \\
(\mathrm{n}=12)\end{array}$ & $\begin{array}{c}\text { HC } \\
(\mathrm{n}=12)\end{array}$ & P-value \\
\hline BMI $\left(\mathrm{kg} \mathrm{m}^{-1}\right)$ & $23.2 \pm 3.0$ & $24.0 \pm 3.1$ & $22.9 \pm 3.6$ & $23.1 \pm 2.7$ & 0.794 \\
$W C>80 \mathrm{~cm}^{2}(n)$ & 8 & 9 & 8 & 6 & \\
VF $>100 \mathrm{~cm}^{2}(n)$ & 4 & 3 & 6 & 4 & \\
\hline
\end{tabular}

Abbreviations: $B M I$, body mass index; $C D$, celiac disease; $D M$, type 1 diabetes mellitus; DMCD, type 1 diabetes mellitus and celiac disease; $\mathrm{HC}$, healthy control; VF, visceral fat area; WC, waist circumference; $M / F$, male/ female. $P$-value: analysis of variance. Values are means \pm s.d. with osteopenia by densitometry: one patient of DM group and other two female participants, postmenopausal, aged 48 and 57 years old. One of these belonged to the control group and one to $\mathrm{CD}$ group, reflecting that almost all patients in all groups were within the normal range for Z-score in the bone mineral density, without any statistical difference.

Results of psychological well-being assessment estimated by the SF-36 questionnaire showed a lower score for functional capacity, physical limitations, pain and social aspects in the $D M$ and CD groups, but without reaching statistical difference (Table 5). Two domains of the SF-36 reached statistically significant differences between DM and healthy control groups: general health $(P=0.042)$ and energy/vitality $(P=0.012)$. Of particular note was the negative correlation between glycemic control, assessed by glycated hemoglobin levels of the DM group, with the domain energy/vitality (Table 6).

Complications associated with T1DM were not correlated with a significant difference in health status $(P=0.22)$, vitality $(P=0.22)$, pain $(P=0.27)$, mental health $(P=0.30)$, but the domain related to emotional limitations attained a statistically significant result $(P=0.00031)$.

QoL was also correlated with compliance to a GFD assessed by the CDAT questionnaire and dosage of autoantibodies for those patients with $C D$. Scores were similar in both DMCD and CD groups $(P=0.688)$. There was good correlation between the positivity of antibody anti-EMA and poor adherence to the GFD ( $P=0.0381$, Fisher's exact test), but without repercussions on QoL. In short, it is possible to conclude that each disease has its own impact in QoL, and the association of both diseases did not result in an important worsening of it.

\section{DISCUSSION}

Our data show that nutritional status and BMI analysis were within the normal range, despite the fact that mean waist circumference was higher than reference values in 53\% of participants in DMCD, $D M$ and $C D$ groups. In the same way, dual energy X-ray absorptiometry results did not show significant differences on visceral fat area or percentage of body fat among the groups

Table 3. Laboratory measurements of vitamins, minerals, and serum and urinary laboratory biomarkers of bone formation and remodeling and bone mineral density

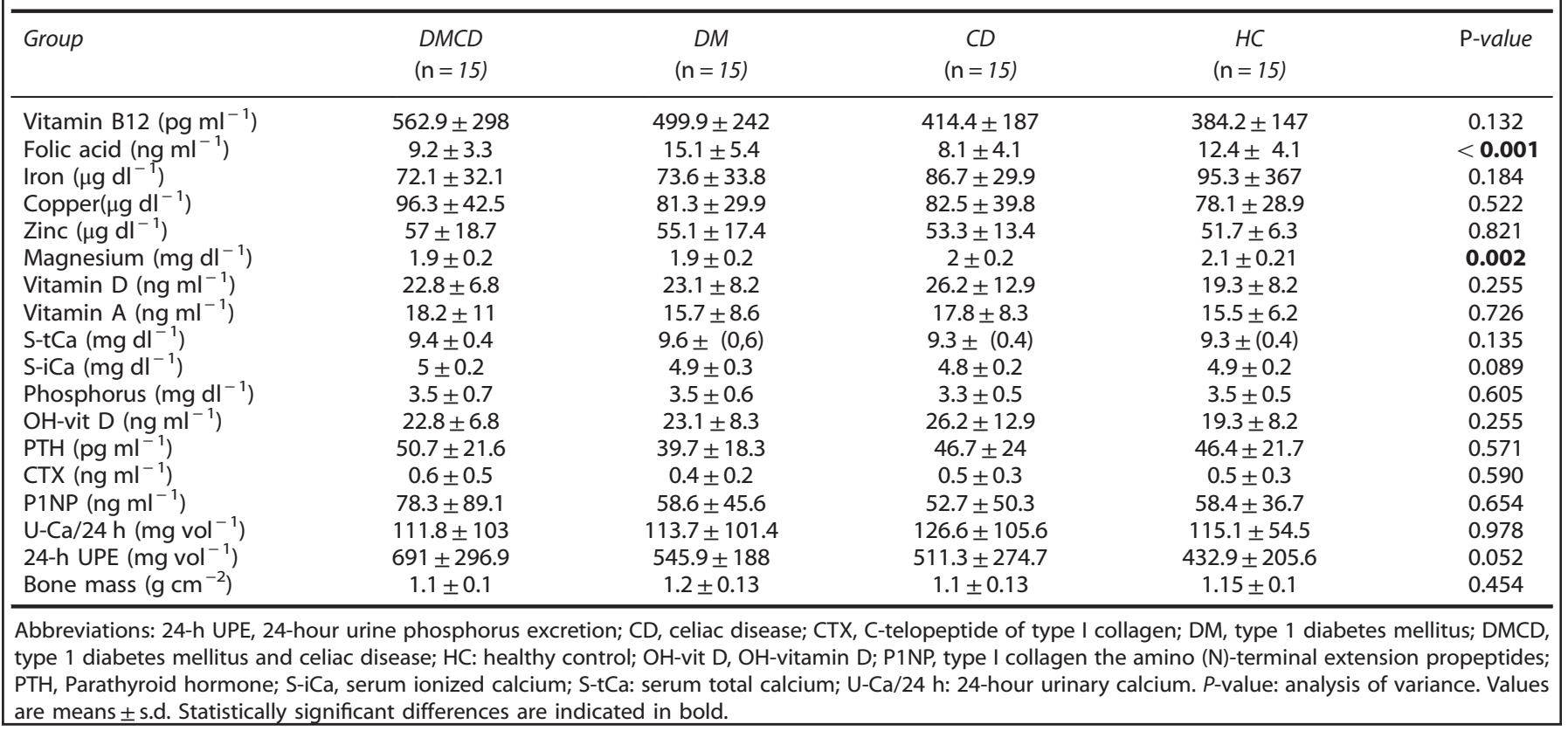


evaluated, although we clearly observed an increase in visceral fat area in those in the CD group. Pitoco et al. ${ }^{21}$ evaluated 120 patients with very similar characteristics to individuals recruited for this study and observed greater severity of subclinical atherosclerosis in DMCD subgroup than in individuals with type 1 diabetes or $C D$ alone. Therefore, they suggested that the

\begin{tabular}{|c|c|c|c|c|c|}
\hline \multirow[t]{2}{*}{ Groups compared } & \multirow[t]{2}{*}{ Mean difference } & \multirow[t]{2}{*}{ s.e. } & \multirow[t]{2}{*}{ P-value } & \multicolumn{2}{|c|}{$\mathrm{Cl}(95 \%)$} \\
\hline & & & & Lower & Upper \\
\hline \multicolumn{6}{|l|}{ Folic acid } \\
\hline DMCD vs DM & -5.96 & 1.58 & 0.002 & -10.29 & -1.63 \\
\hline DMCD vs CD & 1.13 & 1.58 & $>0.999$ & -3.20 & 5.46 \\
\hline DMCD vs GC & -3.17 & 1.58 & 0.302 & -7.50 & 1.16 \\
\hline DM vs CD & 7.09 & 1.58 & $<0.001$ & 2.76 & 1.42 \\
\hline DM vs GC & 2.79 & 1.58 & 0.499 & -1.54 & 7.12 \\
\hline CD vs GC & -4.29 & 1.58 & 0.053 & -8.62 & 0.04 \\
\hline \multicolumn{6}{|l|}{ Magnesium } \\
\hline DMCD vs DM & 0.02 & 0.06 & $>0.999$ & -0.15 & 0.19 \\
\hline DMCD vs CD & -0.11 & 0.06 & 0.421 & -0.28 & 0.06 \\
\hline DMCD vs GC & -0.20 & 0.06 & 0.010 & -0.37 & -0.04 \\
\hline DM vs CD & -0.14 & 0.06 & 0.186 & -0.31 & 0.03 \\
\hline DM vs GC & -0.23 & 0.06 & 0.003 & -0.40 & -0.06 \\
\hline$C D$ vs GC & -0.09 & 0.06 & 0.886 & -0.26 & 0.08 \\
\hline
\end{tabular}

Abbreviations: $\mathrm{BMI}$, body mass index; $\mathrm{CD}$, celiac disease; $\mathrm{Cl}$, confidence interval; DM, type 1 diabetes mellitus; $D M C D$, type 1 diabetes mellitus and celiac disease; HC, healthy control. P-value: Bonferroni test. Statistically significant differences are indicated in bold. association of these two autoimmune diseases (T1DM and CD) could accelerate atherosclerosis.

Dietary deficiencies are common at diagnosis and over active $C D$, especially of folate and vitamins B12 and B6, due to the loss of protein and brush border enzymes in the proximal portions of the intestine, even in the absence of gastrointestinal symptoms. ${ }^{22}$ Rarely are gluten-free products fortified with folic acid and vitamins, distinct from what happens with regular food, and this could be an explanation why approximately one-half of individuals with $C D$ followed up for 10 years persisted with vitamin deficiencies, even with adequate gluten restriction. ${ }^{23,24}$ We found folic acid deficiency in the celiac patients enrolled in this study (DMCD and CD groups). They had between 6 and 12 years of diagnosis with good adhesion to the GFD and steady metabolic control. In contrast, the detection of lower serum magnesium concentrations in the DMCD and DM groups appeared to be an isolated finding. The effect of the association of T1DM and CD on folic acid deficiency and other hydrosoluble vitamins, as well the mechanisms responsible for this deficiency, are unknown. Nonetheless, the involvement of dietary deficiencies or changes in absorption and increased urinary excretion in the face of sustained hyperglycemia may play a role in $\mathrm{it.}^{25}$ However, published data indicate a potential link between magnesium deficiency, and cardiovascular risk factors and atherosclerosis, since magnesium is a natural calcium channel blocker on vascular smooth muscle and myocardium. ${ }^{26}$ Thus, a periodic laboratory evaluation could be advisable to identify individuals, who are deficient and therefore most likely to benefit from supplementation of oligoelements and vitamins.

Neither T1DM nor CD by itself or in association influenced laboratorial parameters of bone metabolism or BMD. This finding can be explained by the characteristics of the population evaluated in this study such as: (1) young adults with normal

Table 5. Result of eight domains of health status questionnaire SF-36 by group

\begin{tabular}{lcccc}
\hline Group & DMCD & $D M$ & $C D$ & HC \\
\hline Physical functioning & $89 \pm 15$ & $76 \pm 23$ & $73 \pm 28.7$ & $88.7 \pm 9$ \\
Physical role limitations & $70 \pm 39.2$ & $61.7 \pm 38.8$ & $65 \pm 39.9$ & $90 \pm 15.8$ \\
Bodily pain & $72.5 \pm 28.1$ & $62.1 \pm 27.4$ & $62.1 \pm 27.6$ & $71 \pm 20.2$ \\
General health perceptions & $47.5 \pm 15.4$ & $45.3 \pm 19.2$ & $57.4 \pm 19.4$ & 0.066 \\
Energy/vitality & $55 \pm 19.2$ & $47 \pm 24.3$ & $62.7 \pm 20.8$ & 0.127 \\
Social functioning & $79.2 \pm 23.5$ & $62.5 \pm 24.5$ & $64.2 \pm 30.2$ & $71.7 \pm 18$ \\
Emotional role limitations & $53.3 \pm 43.3$ & $51.1 \pm 48.6$ & $68.9 \pm 38.8$ & $792 \pm 20$ \\
Mental health & $61.9 \pm 23.8$ & $54.9 \pm 22.6$ & $68.3 \pm 20.4$ & $77.8 \pm 30$ \\
\hline
\end{tabular}

Abbreviations: $C D$, celiac disease; DM, type 1 diabetes mellitus; DMCD, type 1 diabetes mellitus and celiac disease; $H C$, healthy control. ${ }^{a}$ statistically significant differences between DM and HC groups; $n=15$ patients per group. $P$-value: analysis of variance test. The value for each domain of the questionnaire of quality of life ranges from 0 (the worst state health) to 100 (the best state health). Statistically significant differences are indicated in bold.

Table 6. Relationship among A1c and domains of health status questionnaire SF-36 in T1DM patients

\begin{tabular}{|c|c|c|c|c|c|c|}
\hline & Correlation & P-value & $\mathrm{n}$ & Correlation & P-value & $\mathrm{n}$ \\
\hline Physical role limitations & 0.049 & 0.861 & 15 & 0.115 & 0.684 & 15 \\
\hline Bodily pain & -0.054 & 0.849 & 15 & 0.368 & 0.177 & 15 \\
\hline General health perceptions & 0.091 & 0.748 & 15 & 0.423 & 0.116 & 15 \\
\hline Emotional role limitations & 0.122 & 0.666 & 15 & 0.063 & 0.822 & 15 \\
\hline Mental health & 0.239 & 0.391 & 15 & 0.238 & 0.393 & 15 \\
\hline
\end{tabular}

Abbreviation: T1DM, type 1 diabetes mellitus. P-value: Pearson product-moment correlation coefficient. Statistically significant differences are indicated in bold. 
weight; (2) majority of women in reproductive age; (3) similar intake of calcium and vitamin D; (4) regular glycemic control, thus without chronic urinary calcium excretion; and (5) celiac patients with good adhesion to GFD, which somehow contributed to the formation of adequate bone mass or did not impact it negatively.

The analysis of the SF-36 questionnaire identified that patients with DM1 achieved lower scores than the control group in general health and vitality areas, suggesting that 'having diabetes' requires more attention to lifestyle habits and daily treatment, resulting in an increased perception of the disease. Probably the complexities involved in managing the treatment influenced the QoL of these individuals. In addition, the presence of diabetes-related complications was associated with lower scores on domain role limitations due to emotional problems. Likewise, in a cohort of patients with T1DM using multiple doses of insulin, van Dijk et al. ${ }^{10}$ found a decrease in scores on the assessment by the SF-36 questionnaire and EuroQol-VAS, interpreted as resulting from the complexity of treatment.

The WESDR (Wisconsin Epidemiologic Study of Diabetic Retinopathy) evaluated the QoL in individuals with T1DM 10 years after the first phase of the study and concluded that the development of complications, especially cardiovascular diseases, as well as changes in employment (retirement, occupying lower positions and unemployment) were associated with worsening of QoL. ${ }^{27}$ Interestingly, we observed the presence of complications did not affect domains of QoL potentially related to limitations of the physical functional capacity or pain, but they had a major impact on the field concerning emotional limitations. Moreover, we observed a higher prevalence of complications in DM than in DMCD group, in spite of the similar duration of the disease.

Data published by Bakker et al. ${ }^{3}$ pointed out a significant negative impact on QoL related to concerns inherent to diabetes and social fears of adults with both diseases. Nevertheless, we observed that individuals with both diseases (DMCD) tended to achieve higher QoL scores, which means better general health when compared with DM, CD or control subjects. These results also diverge from data published by Hallert et al. ${ }^{28}$ that showed that $C D$ patients had more difficulty in living with the disease than those with diabetes or the general population. They referred to a feeling of 'being excluded' from the social context due to the reduced availability of gluten-free foods. This difference could be related to either good adherence to both treatments or to the fact that patients with difficulties in adapting to treatment were more permissive to food transgressions, omissions in insulin application and less worried about glycemic control. Therewith, they had SF-36 scores closer to healthy controls, without the perception of being sick or influenced by the disease in social life.

The limitation of this study is related to the sample size. The inclusion of only 15 individuals in each group could have influenced the lack of statistical significance of waist circumference data, the compartmentalized analysis of the total body weight or even other parameters related to QoL. However, as the group was matched for the presence of each disease with healthy controls, the analysis of nutritional health and QoL could be considered representative for young adults, since there is a low prevalence of the association of both diseases in the general population. Likewise, other studies have been published with small sample size and the authors also reported the difficulty in recruiting patients with T1DM and CD. ${ }^{29-33}$

Recently a multicenter randomized controlled trial (CD-DIET) ${ }^{34}$ has been designed to clarify the impact of a GFD on clinically relevant outcomes as metabolic control, bone health, glycemic variability and QoL in children and adults with type 1 diabetes and asymptomatic $C D$, making clear the need for specific studies directed to this population. In this sense, the importance of this study was to point out that T1DM associated with CD was not related to worsening QoL, nor did it have a negative impact on glycemic control in a population of individuals matched for age and gender with others, who have just one of these diseases and share similar social environment. This information seems to be valuable to understand the health-disease process and to contribute to the re-evaluation of promotion, prevention, treatment and rehabilitation of the patient's health.

\section{CONFLICT OF INTEREST}

The authors declare no conflict of interest.

\section{REFERENCES}

1 Fasano A, Berti I, Gerarduzzi T, Not T, Colletti RB, Drago S et al. Prevalence of celiac disease in at-risk and not-at-risk groups in the United States: a large multicenter study. Arch Intern Med 2003; 163: 286-292.

2 Association AD. 3. Foundations of care and comprehensive medical evaluation Diabetes Care 2016; 39: S23-S35.

3 Bakker SF, Pouwer F, Tushuizen ME, Hoogma RP, Mulder CJ, Simsek S. Compromised quality of life in patients with both type 1 diabetes mellitus and coeliac disease. Diabet Med 2013; 30: 835-839.

4 Wild D, Robins GG, Burley VJ, Howdle PD. Evidence of high sugar intake, and low fibre and mineral intake, in the gluten-free diet. Aliment Pharmacol Ther 2010; 32: 573-581.

5 Thompson T, Dennis M, Higgins LA, Lee AR, Sharrett MK. Gluten-free diet survey: Are Americans with coeliac disease consuming recommended amounts of fibre, iron, calcium and grain foods? J Hum Nutr Diet 2005; 18: 163-169.

6 Kinsey L, Burden ST, Bannerman E. A dietary survey to determine if patients with coeliac disease are meeting current healthy eating guidelines and how their diet compares to that of the British general population. Eur J Clin Nutr 2008; 62 1333-1342.

7 Hopman EGD, le Cessie S, von Blomberg BME, Mearin ML. Nutritional management of the gluten-free diet in young people with celiac disease in The Netherlands. J Pediatr Gastroenterol Nutr 2006; 43: 102-108.

8 Churruca I, Miranda J, Lasa A, Bustamante MÁ, Larretxi I, Simon E. Analysis of body composition and food habits of Spanish celiac women. Nutrients 2015; 7: 5515-5531.

9 Brazeau AS, Mircescu H, Desjardins K, Leroux C, Strychar I, Ekoé JM et al. Carbohydrate counting accuracy and blood glucose variability in adults with type 1 diabetes. Diabetes Res Clin Pract 2013; 99: 19-23.

10 Van Dijk PR, Logtenberg SJ, Groenier KH, Keers JC, Bilo HJ, Kleefstra N. Fifteen-year follow-up of quality of life in type 1 diabetes mellitus. World J Diabetes 2014; 5 : 569-576.

11 Rubin RR, Peyrot M. Quality of life and diabetes. Diabetes Metab Res Rev 1999; 15 205-218.

12 Nachman F, Mauriño E, Vázquez H, Sfoggia C, Gonzalez A, Gonzalez V et al. Quality of life in celiac disease patients. Prospective analysis on the importance of clinical severity at diagnosis and the impact of treatment. Dig Liver Dis 2009; 41: 15-25.

13 Nepa - Núcleo de Estudos e pesquisas em Alimentação. Tabela Brasileira de Composição de Alimentos - TACO 4a edição revisada e ampliada. 2011:161.

14 Philippi ST. Tabela de Composição Dos Alimentos: Suporte Para Decisão Nutricional 2nd edn. Coronário: São Paulo, 2002.

15 Brasileira de Sociedade. Diretrizes Da Sociedade Brasileira de Diabetes 2015-2016. AC Farmacêutica Ltda: São Paulo, 2016; pp 337.

16 Otten Jennifer J, Hellwig Jennifer Pitzi, Linda D Meyers. Dietary Reference Intakes: The Essential Reference for Dietary Planning and Assessment an Invaluable Resource (Food and Nutrition Board; Institute of Medicine, edn.) The National Academies Press: Washington DC, USA, 2006; pp 560.

17 Institute of Medicine (US) Committee to Review Dietary Reference Intakes for Vitamin D and Calcium. Ross AC, Taylor CL, Yaktine AL, Del Valle HB (eds). Dietary Reference Intakes for Calcium and Vitamin D. National Academies Press: Washington, DC, USA, 2011.

18 Ciconelli Ferraz, MB, Santos W, Meinão I, Quaresma M. Tradução para a língua portuguesa e validação do questionário genérico de avaliação de qualidade de vida SF-36 (Brasil SF-36). Rev Bras Reum 1999; 39: 143-150.

19 Leffler DA, Dennis M, Edwards George JB, Jamma S, Magge S, Cook EF et al. A simple validated gluten-free diet adherence survey for adults with celiac disease. Clin Gastroenterol Hepatol 2009; 7: 530-536.

20 World Health Organization. Waist Circumference and Waist-Hip Ratio : Report of a WHO Expert Consultation, Geneva, 8-11 December 2008, vol 39.World Health Organization: Geneva, Switzerland, 2011.

21 Pitocco D, Giubilato S, Martini F, Zaccardi F, Pazzano V, Manto A et al. Combined atherogenic effects of celiac disease and type 1 diabetes mellitus. Atherosclerosis 2011; 217: 531-535 
22 Wierdsma NJ, van Bokhorst-de van der Schueren MAE, Berkenpas M, Mulder CJJ, van Bodegraven AA. Vitamin and mineral deficiencies are highly prevalent in newly diagnosed celiac disease patients. Nutrients 2013; 5: 3975-3992.

23 Thompson T. Folate, iron, and dietary fiber contents of the gluten-free diet. J Am Diet Assoc 2000; 100: 1389-1396.

24 Hallert C, Grant C, Grehn S, Grännö C, Hultén S, Midhagen G et al. Evidence of poor vitamin status in coeliac patients on a gluten-free diet for 10 years. Aliment Pharmacol Ther 2002; 16: 1333-1339.

25 Sales $\mathrm{CH}$, Pedrosa LFC. Magnesium and diabetes mellitus: Their relation. Clin Nutr 2006; 25: 554-562.

26 Shah NC, Shah GJ, Li Z, Jiang XC, Altura BT, Altura BM. Short-term magnesium deficiency downregulates telomerase, upregulates neutral sphingomyelinase and induces oxidative DNA damage in cardiovascular tissues: relevance to atherogenesis, cardiovascular diseases and aging. Int J Clin Exp Med 2014; 7: 497-514.

27 Hirai FE, Tielsch JM, Klein BEK, Klein R. Ten-year change in self-rated quality of life in a type 1 diabetes population: Wisconsin Epidemiologic Study of Diabetic Retinopathy. Qual Life Res 2013; 22: 1245-1253.

28 Hallert C, Sandlund O, Broqvist M. Perceptions of health-related quality of life of men and women living with coeliac disease. Scand J Caring Sci 2003; 17: 301-307.

29 Tsouka A, Mahmud FH, Marcon MA. Celiac disease alone and associated with type 1 diabetes mellitus. J Pediatr Gastroenterol Nutr 2015; 61: 297-302.

30 Amin R, Murphy N, Edge J, Ahmed ML, Acerini CL, Dunger DB. A longitudinal study of the effects of a gluten-free diet on glycemic control and weight gain in subjects with type 1 diabetes and celiac disease. Diabetes Care 2002; 25: 1117-1122.

31 Hansen D, Brock-Jacobsen B, Lund E, Bjørn C, Hansen LP, Nielsen C et al. Clinical benefit of a gluten-free diet in type 1 diabetic children with screening-detected celiac disease: a population-based screening study with 2 years' follow-up. Diabetes Care 2006; 29: 2452-2456.

32 De Melo EN, Deda L, Har R, Reich HN, Scholey JW, Daneman D et al. The urinary inflammatory profile in gluten free diet-adherent adolescents with type 1 diabetes and celiac disease. J Diabetes Complications 2016; 30: 295-299.

33 Zhao Z, Miao D, Michels A, Steck A, Dong F, Rewers M et al. A multiplex assay combining insulin, GAD, IA-2 and transglutaminase autoantibodies to facilitate screening for pre-type 1 diabetes and celiac disease. J Immunol Methods 2016; 430: 28-32.

34 Mahmud Farid H, De Melo Emilia N, Noordin Karima, Assor Esther, Sahota Kamaljeet, Davies-Shaw Jolie et al. 'The Celiac Disease and Diabetes-Dietary Intervention and Evaluation Trial (CD-DIET) Protocol: a randomised controlled study to evaluate treatment of asymptomatic coeliac disease in type 1 diabetes.'. BMJ Open 2015; 5: e008097.

This work is licensed under a Creative Commons Attribution 4.0 International License. The images or other third party material in this article are included in the article's Creative Commons license, unless indicated otherwise in the credit line; if the material is not included under the Creative Commons license, users will need to obtain permission from the license holder to reproduce the material. To view a copy of this license, visit http://creativecommons.org/licenses/ by/4.0/

(c) The Author(s) 2017 